\title{
Growth Model for Filamentary Streamers in an Ambient Field
}

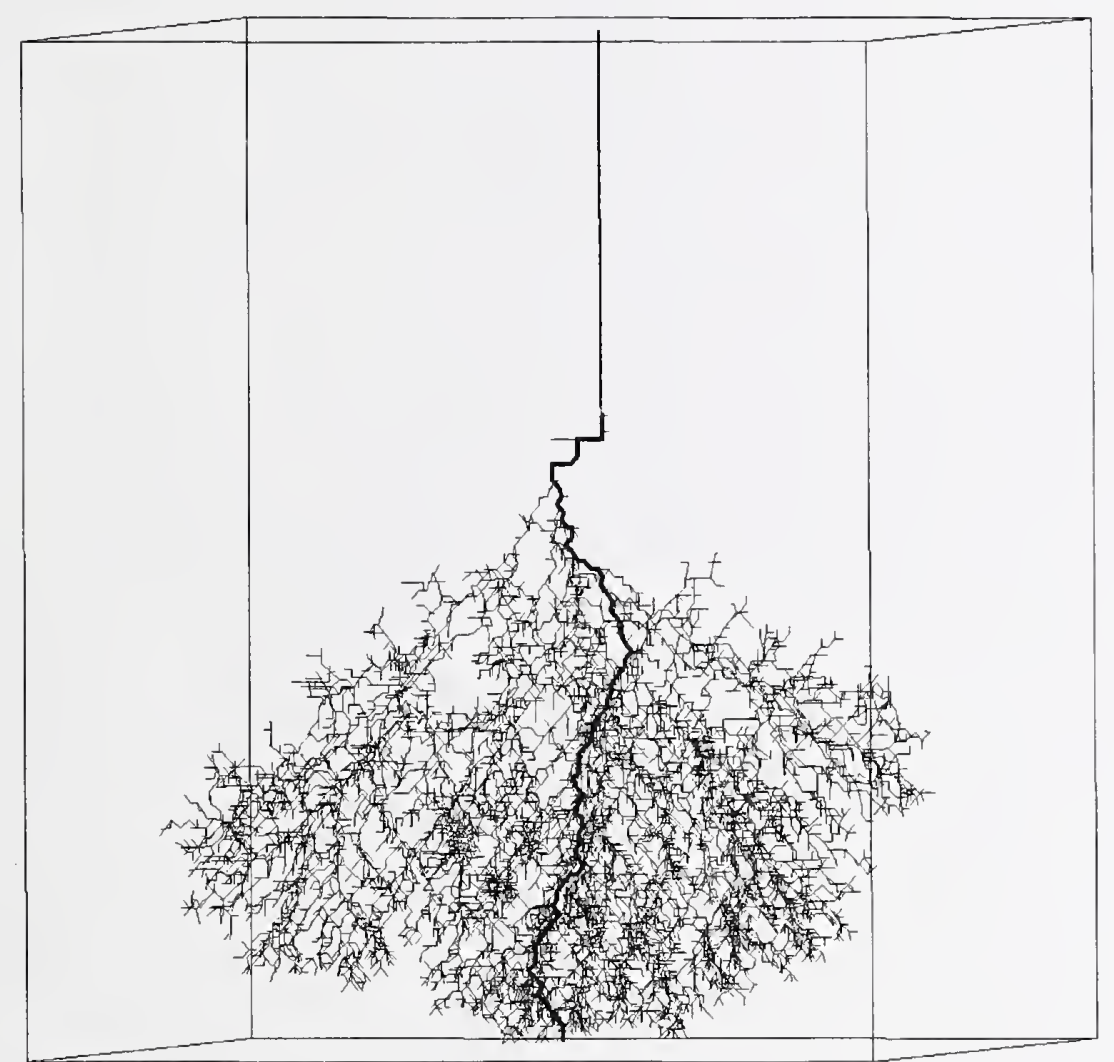

\section{H.A. Fowler \\ J.E. Devaney J.G. Hagedorn}

U.S. DEPARTMENT OF COMMERCE Technology Administration High Performance Systems and Services Division National Institute of Standards and Technology

Gaithersburg, MD 20899

April 2000

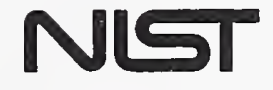

National Institute of Standards and Technology Technology Administration U.S. Department of Commerce

10.6504 



\section{NISTIR 6504}

\section{Growth Model for Filamentary Streamers in an Ambient Field}

\section{H.A. Fowler \\ J.E. Devaney J.G. Hagedorn}

U.S. DEPARTMENT OF COMMERCE Technology Administration High Performance Systems and Services Division National Institute of Standards and Technology

Gaithersburg, MD 20899

April 2000

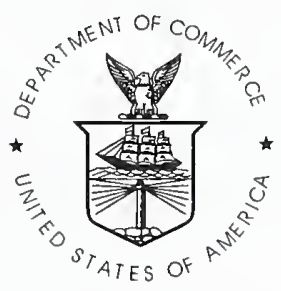

U.S. DEPARTMENT OF COMMERCE William M. Daley, Secretary

TECHNOLOGY ADMINISTRATION

Dr. Cheryl L. Shavers, Under Secretary of Commerce for Technology

NATIONAL INSTITUTE OF STANDARDS

AND TECHNOLOGY

Raymond G. Kammer, Director 



\title{
Growth Model for Filamentary Streamers in an Ambient Field
}

\author{
H.A. Fowler, J.E. Devaney, and J.G. Hagedorn \\ High Performance Systems and Services Division \\ National Institute of Standards and Technology, Gaithersburg, MD 20899
}

\begin{abstract}
We have simulated the fast streamer stage of liquid dielectric breakdown as stochastic growth of a branching fractal tree. Breakdown and threshold properties of the fluid are represented in the random filter procedure. A range of fractal densities, from sparse to bushy, is approximated by the choice of power-law (4th-power to linear). The choice of threshold (cutoff) voltage also significantly affects the growth form. These parameters combine with the shape and concentration of the electric field, to regulate the distribution and directedness of the local discharge growth pattern. Inclusion of a voltage gradient along the streamer tree produces a secondary narrowing effect on the growth.
\end{abstract}

A large grid (128 cubed) is used for the discretization. Diagonal growth paths to neighbor-vertices are included, increasing the choice of available directions for each discharge event.

We use a combination of data-parallel programming and three-dimensional visualization. Complete growth histories, evolving from the voltage distribution, can be displayed in animation or in color banding against the "trials" variable, which simulates a time tick. Side views of the structures provide comparison against sub-microsecond snapshots from experiment. Results include sparse, directed trees evolving from a 4th-power-law filter; also dense trees from a linear filter, whose conical upper-envelope boundary is strongly influenced by the choice of threshold (cutoff) potential.

\section{Introduction}

Filamentary streamers evolve rapidly in a surrounding voltage field, which influences their shape and density. We explore a simplified "stochastic Laplacian fractal" simulation for this phenomenon [1]. We do not include microscopic details of the physical processes. Our purpose is to capture the global features of the growth.
Elements of the numerical algorithm are as follows:

- Assume the streamer tree is conductive (either with or without an internal voltage gradient), and attached to the starting electrode.

- Solve Laplace's equation throughout the full interior region of the liquid dielectric. Boundary conditions are set by the starting electrode plus the tree (a growing, fractal object) at one polarity; and the counterelectrode, which is assigned the opposite-polarity voltage of 1.0.

- Examine neighbor sites one grid-step away from the tree. Where voltage difference between the site and the tree exceeds a sharp threshold level, then compare against random numbers by a method which yields a weighted distribution of survivors. Attach these survivors to the tree.

- Cycle these two steps until the counterelectrode is reached. Since the tree forms one boundary condition for the electrostatic field, the voltage field is redistributed after each growth stage, and must be recalculated. This give-and-take between the ambient field and new streamer links dominates the growth process.

- Record grid position for each new growth step, together with the directional index for its link (26 directions are possible) and the count number of the statistical trial. Thus the distribution, directions, and rate of growth are followed in time, as they are controlled by the evolving voltage field.

- Display the growth form in three dimensions. Rotation, color banding, and animation can be used to clarify the growth history.

In these simulations, the probability weighting for survivors is taken to be a power of the voltage difference. Figure 1, which is a $\log$-log plot of breakdown probability, $P\left(V_{i}\right)$ versus neighbor-site voltage difference, $V_{i}$, sketches the assumed response law for 

differing powers. By varying the weighting powerlaw exponent from 4 to 1 we demonstrate below that the increasing visual bushiness (sparse to dense) results from the spread of forward directional concentration, about the axes of the individual growth tips.

High geometric resolution, detailed threedimensional calculation of the voltage field, and a plausible method for estimating time progression are necessary. As Biller has noted [2], length, voltage, and time must be explicitly scaled in the discretized model to approximate a history of the physical growth, which is more than a geometrical abstraction.

The model is non-specific with regard to the microscopic mechanism of streamer growth. It does not treat the microscopic electric field strength at the tip surface. Neighbor-voltage values are taken at a common radius (one grid step length, corresponding roughly to a $30-50$ micrometer segment) from the tip; they are considered to be proportional to the vector electric field strength at the neighbor sites. The electric field at the tip point (observed in experiment to have radius 3 micrometers or less) is of course very much higher - sufficiently strong to produce cascade ionization of the dielectric fluid. Here the neighbor-voltage values are used only as directional probability indicators. (For statistical uniformity, each diagonal-step voltage is down-weighted by a $1 / \mathrm{r}$ factor.)

\section{Probability Model}

The shape and concentration of the neighbor-voltage distribution, and the slope of the selected breakdown law affect the rate, the distribution, and the directionality of the modeled growth at each stage. Dissado et al [3] have shown that for an extended projecting tip the ratio of axial to lateral field magnitude in three dimensions is roughly 1.22:1. For a linear-law survivor probability this regulates the rates of forward vs. lateral growth. For square-law, cube-law, and fourth-power cases the ratio would change to $1.49: 1,1.82: 1$, and $2.22: 1$ respectively. These ratios affect the extent of splitting and the bushy-to-sparse appearance.

The power-law lines for different exponents are sketched in Figure 1. These correspond to nominal response curves for the fluid. Ordinate is the probability (or rate) of breakdown; the abscissa is the voltage difference between the tree and the neighbor site. Raising the exponent exerts an axial constriction on the local growth cone, concentrating growth ahead of the tip [3]. Lowering the exponent gives rise to a more dense, spread pattern of twig growth in a more rounded frontal zone. This effect is seen more clearly when diagonal growth paths are included.

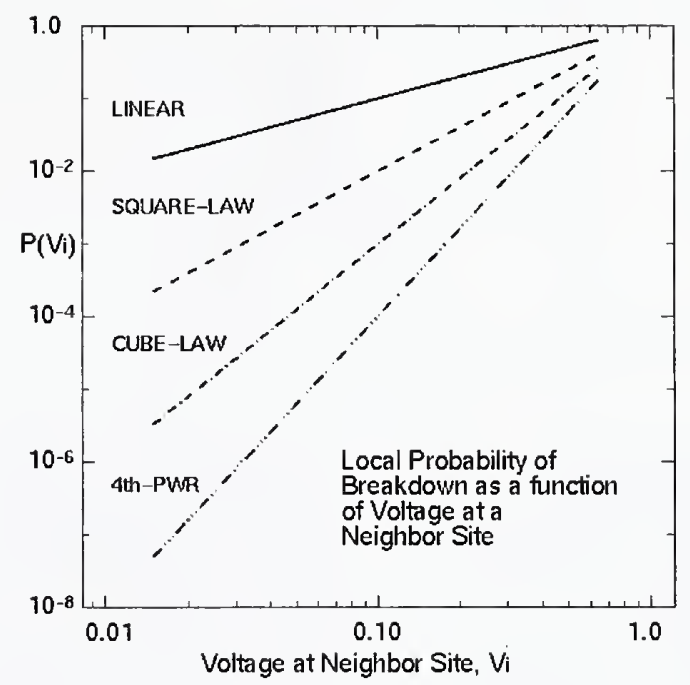

Figure 1. Local breakdown probability (for a link discharge), $\mathrm{P}\left(V_{i}\right)$ as a function of neighbor-site voltage. Scales are $\log -\log$. Choice of power-law exponent represents the nominal response of the fluid. Threshold (cutoff) parameter determines where the left end of each line drops to zero. Voltage distributions overlap this dropoff point.

The vertical-scale level of each graph is arbitrary, and corresponds to the rate (or probability) of the physical process producing the growth. In practice, streamers showing the cubic and 4th-power "sparse" structure grow very rapidly relative to the growth speed of the more dense, space-filling filamentary streamers produced by a linear-law filter. Thus, the corresponding experimental plot might show the linear-growth line at a lower level on the graph.

Varying the threshold (cutoff) voltage parameter also has a noticeable effect on the overall density of discharged links. (The lines in Fig. 1 are limited by a drop to zero at their left end, which is positioned by this choice.) As we observe below, the choice of a threshold value controls the conical sector angle into which the dense linear-weighted fractal tree can spread.

Clocking of Monte Carlo time is considered in this model; thus the distribution, directions, and rate of 

growth are followed together, as they are controlled by the evolving voltage field. In the examples we give, the "Monte Carlo" time tick is extremely short - each growth-stage interval is only a hundredth or less of the overall growth duration, corresponding therefore to a real time interval of the order of nanoseconds. In cases of very low probability, as on the lower half of Figure 1, the "waiting time" between events is estimated by the BKL method[4].

Display is carried out in three dimensions. On a work station, color banding can be used to mark the time history of growth; dynamic rotation of the image is extremely useful in distinguishing details of the growth form. Animation of frames against the time-tick variable is also readily achieved.

In the figures below, simulation has been performed on a $128 \times 128 \times 128$ grid. The top surface of the cube represents the starting electrode, with an attached central needle. The lower surface is the counterelectrode; side boundary conditions are periodic. Unless otherwise indicated, as in Figure 8, the streamer growths are assumed to be perfectly conducting, without a retained voltage gradient. The heavy line denotes the connecting path, or "leader" track, along which high-current breakdown will occur.

\section{4th-Power-law}

Figure 2 is a representative case of streamer simulation, modeled with a probability filter which produces a 4th-power-law survivor population. Note that the branches are sparse and forward-directed. The bare-tree appearance results from differential preference for tip extension versus lateral branching. 4th-power-law also tends to maximize the apparent growth speed in the short-gap stages of the path, so that the advance across the second half of the interval occurs in a very small fraction of the total elapsed time. Inclusion of a retained voltage gradient in the streamer tree, when this is set at $0.30 \times$ the voltage difference causing breakdown on the individual link, has no noticeable effect on the general appearance of this sparse streamer form.

Experimental streamers, of similar sparse appearance, can be identified in recent results by Miyano et al. [6], which describe positive streamer propagation in perfluoro liquids.

Tentatively, we would identify this form of sparse, forward-directed growth with the very fast "type

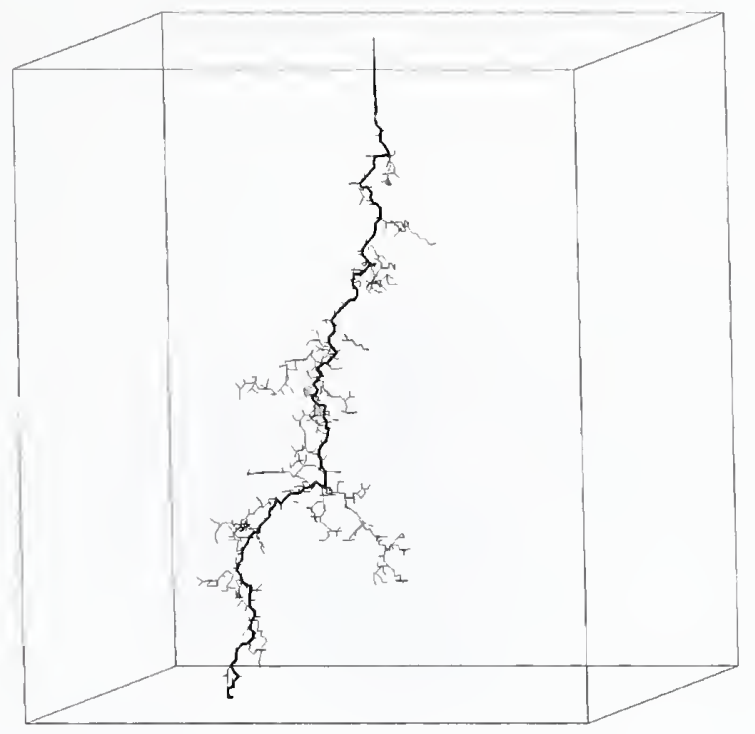

Figure 2. 4th-power-law example. Attached central needle has length fifteen grid steps. The threshold (cutoff) voltage is set at 0.0700 , just slightly below the largest voltage on a neighbor site to the needle. 20089 (est.) statistical tries have produced 956 discharged links. Growth across the second half of the point-to plane gap occurs in about $1 / 16$ of the entire "Monte Carlo" elapsed time. This speedup is a result of the low, screened initial field near the originating electrode, and the liigh power law assumed in the model.

4" streamers first characterized by Hebner [7], and studied in more detail by Massala and Lesaint [8].

\section{Square-law}

Figures 3,4 , and 5 are representative growths obtained with a square-law weighting. In Figure 3 a relatively high threshold (cutoff) voltage has been assumed (0.2000), corresponding to a low overvoltage in experiment. In Figures 4 and 5 this threshold is lowered to 0.1500 and 0.0500 , respectively, to simulate increased overvoltages applied to the electrode gap. Noticeable features are the increase in major side-branching, and the wider envelope shape. The streamer tree has more branches and a generally more dense, bushy appearance, because the relative probability of lateral branching has increased. Individual branches are less tightly forward-directed by the voltage distribution.

Experimental cases having a similar structure are found in results by Lesaint and Massala [9] on positive streamer propagation in large oil gaps. At increasing levels of overvoltage, they find streamer 



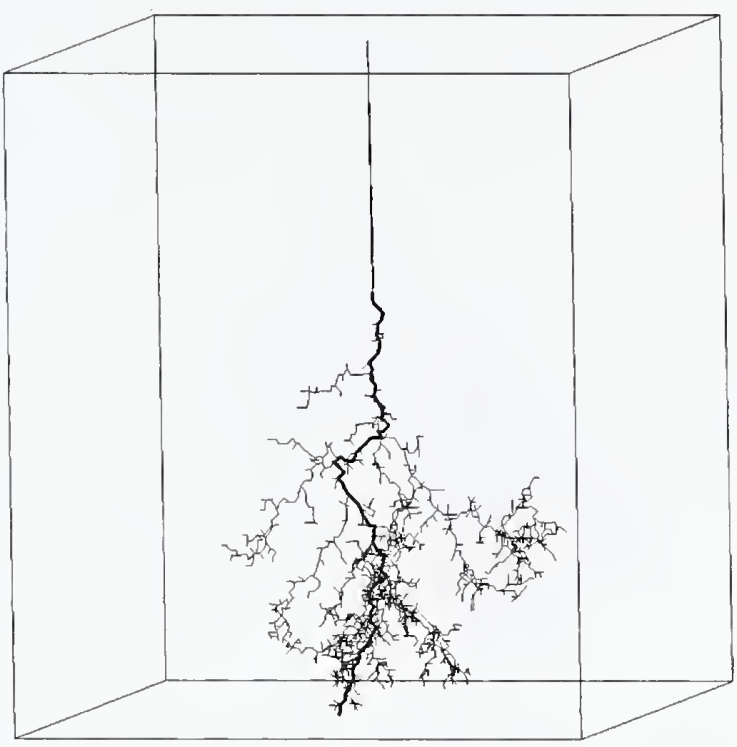

Figure 3. Square-law streamer simulation. 314 statistical tries have given rise to 1832 discharged links. The needle has been lengthened to 47 grid intervals, which more closely simulates the "point-to-plane" configuration. Threshold (cutoff) voltage level is set at 0.2000 , just slightly below the largest neighbor voltage adjacent to the needle tip.

forms with broader branching patterns and more rapid growth.

\section{Linear weighting}

Figures 6 and 7 show the dense, widely-branched structure which a linear-weighted growth filter produces. By contrast with the cube- and square-law cases, these trees tend to divide immediately into several ( 3 or 4 ) large major branches, which spread densely through the volume but remain self-avoiding and singly connected.

Figure 6 evolves from a threshold (cutoff) voltage of 0.1200 , Figure 7 with 0.2000 . The strong influence of this parameter on the conical upper envelope of the dense structure is noted. The effect of electrical screening is pronounced, as the bushy growing front intervenes between the counterelectrode and the early portion of the growth. The upper development of the tree, near the starting electrode, is limited at a roughly conical envelope, where growth has ceased as the voltage field is screened back below the abrupt cutoff value.

A conical envelope, for dense positive streamers in

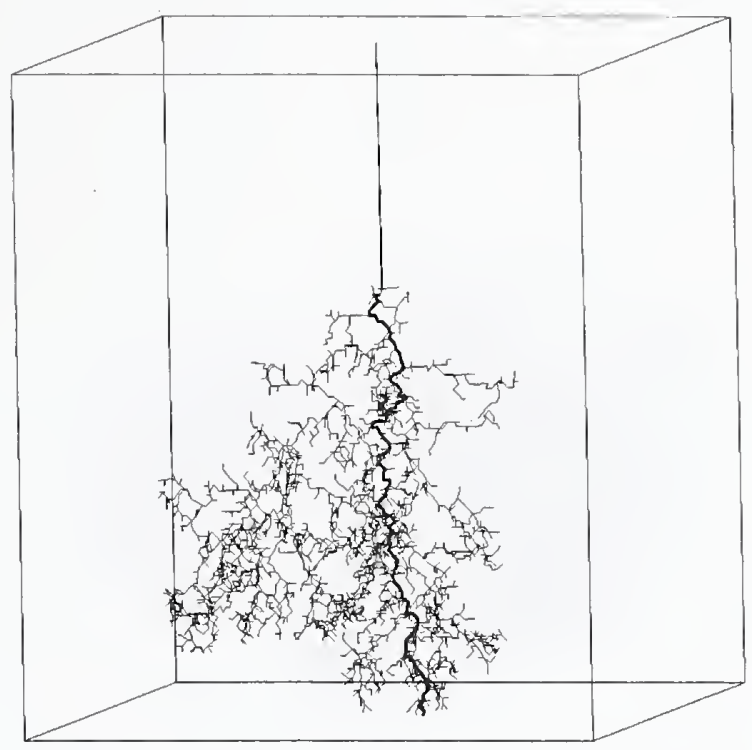

Figure 4. Square-law simulation, with threshold voltage set at 0.1500 . 262 statistical tries have produced 3389 discharged links. Lateral branching density has increased, especially near the origin at the starting needle.

n-hexane, has been noted experimentally by Stricklett et al. [10]. Badent, Kist, and Schwab [11] have observed a constriction of the conical propagation sector, associated with increasing pressure, in experimental positive streamers in insulating oil. Thus, the increase in pressure in their experinent is producing a change which corresponds to the narrowing of the cone, when our threshold (cutoff) parameter is increased. Earlier high-resolution images by Chadband [12] had demonstrated the fine filamentary detail of dense positive streamers.

\section{Effect of Voltage Gradient in the Tree}

A further narrowing and "squeezing" effect can be produced in the model by assuming a retained voltage gradient, from tips to base of the tree, as it grows. In Figure 8 we show the additional effect of assuming that each breakdown leaves a retained voltage gradient in its segment of the tree, equal to 0.40 of the voltage difference causing that segment of the discharge. Noticeable here is the very sparse "trunk" portion starting from the needle. The effect of the introduced gradient is to reduce voltagedifference values between the tree and the candidate neighbor sites, so that the constriction effect of the cutoff potential is more strongly felt.

Massala and Lesaint [13] have reported measure- 



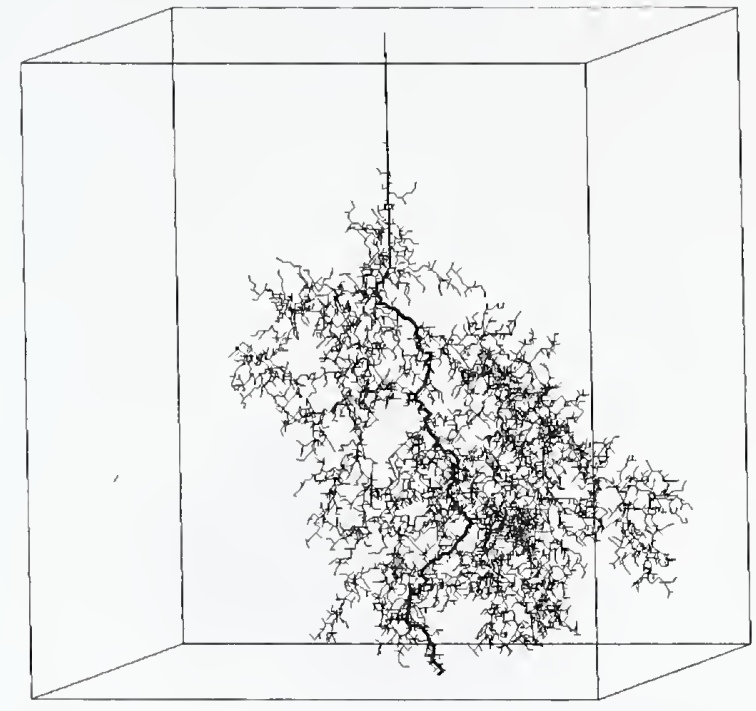

Figure 5. Square-law simulation. Threshold voltage is set at 0.0500 , well below the largest neighbor voltage. 92 statistical tries have produced 7476 discharged links.

ments relating to the voltage drop within streamers. They note that raising the applied voltage may give rise to higher internal conduction, i.e. lower voltage drop inside the streamer.

\section{Overall Growth Rates}

Figure 2 and Figure 6 represent extremes of growth rates. Figure 2, with 4th-power filtering in the shortneedle, "uniform field" configuration, makes a slow start followed by high acceleration across the second half of the gap. Figure 6 , with linear-law filtering in the long-needle, "point-to-plane" configuration, grows as an enlarging, space-filling sphere whose radius increases linearly with time, to a first approximation. In this case the effect of diminishing gap to the counterelectrode is evidently offset by the brush-tip spreading, which reduces local field around individual tips.

Growth rates for individual processes, using squarelaw and cube-law filters, are intermediate between these extreme cases. Introducing a retained voltage gradient appears to have very little effect on rates of forward growth; its principal effect is to constrain the lateral branching.

\section{Summary of Results}

Simplified programming has allowed us to build details into the model in easy steps: high geomet-

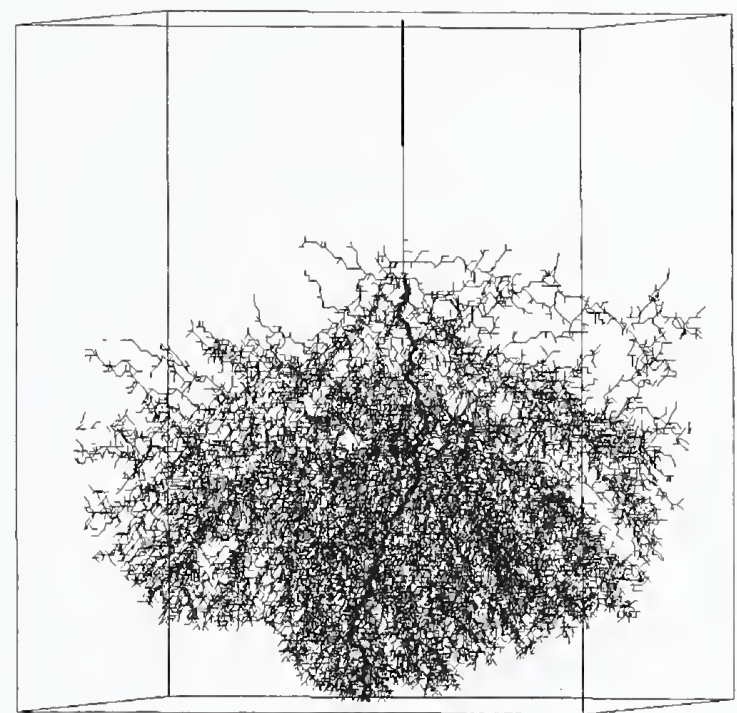

Figure 6. Linear response law. Growth is dense. 91 statistical tries have produced 27,837 discharged links. The front of the growth, facing the counterelectrode, has become rounded and brush-like; this rounding counteracts the field enhancement from the diminished gap distance, so that forward growth proceeds at a nearly constant rate. The upper envelope of growth is a flat cone, almost level with the tip of the needle.

ric resolution on a large grid, inclusion of diagonal paths, a method for scaling time and growth rates, and effect of statistical filters with power laws, voltage thresholds, and a retained voltage gradient in the tree.

The model is put forward as a simplified means for standardized comparisons of different features of streamer behavior. Thus, as its parameters are changed, we have simulated a range of effects with experimental counterparts.

\section{Comparison with Other Models}

Our simulation has several features in common with the recent work of Karpov and Kupershtokh [14], including simultaneous (concurrent) distributed growth, and growth along diagonal links to neighbors. They calculate a "stochastic time", with an intent similar to our "Monte Carlo" time tick. In our case the time tick is directly counted unless the waiting time is very long, in which case it is estimated by a method [4] adapted from molecular mechanics.

Some differences include: 



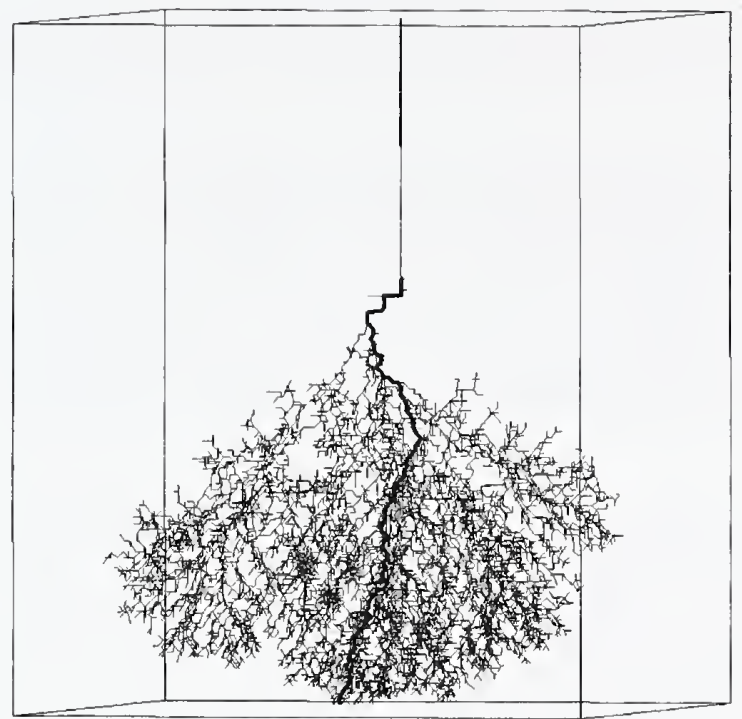

Figure 7. Linear filter. The threshold (cutoff) voltage has been raised to 0.200 . Branches next to the narrower conical upper envelope show a more sparse appearance than the dense structure approaching the cathode. Total number of discharged links has been reduced to 10,000 , in 127 statistical tries. The cutoff from screening is having a pronounced narrowing effect on the conical sector of propagation.

- They assume an ohmic potential drop from tips to base along the streamer tree. In the absence of a clear experimental determination of the factors which set this voltage gradient, we have arbitrarily simulated it as an adjustable fraction of the individual voltage drop causing breakdown in each link. Experiments by Badent and colleagues have shown the possibility of a lengthwise internal voltage gradient.

- They assume a spherical outer boundary condition about a starting point, with the tree growing as a ball. They find the local fractal dimension declining as the tips reach larger radii. Our simulation, confined between electrode plates, shows some increase in local fractal density, as the tips progress.

- They assume a statistical spread of lower threshold (cutoff) values; whereas we take a sharp threshold [5].

R. Badent [15] has presented a detailed theoretical sketch of possible electron-transport modes within the streamer. Tentatively, he identifies each ob-

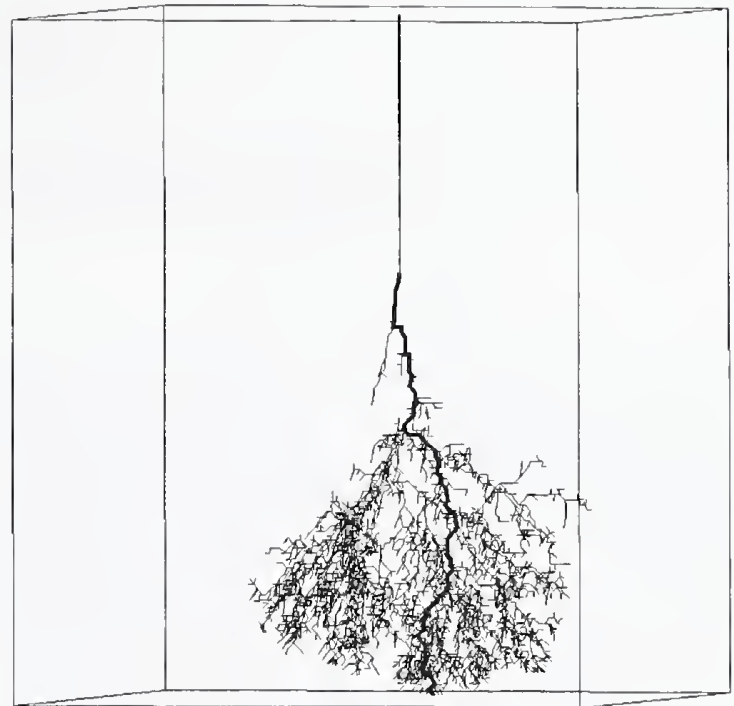

Figure 8. Linear filter. With the threshold voltage remaining at 0.200 , a retained internal voltage gradient has been added throughout the growth of the streamer, the gradient across each segment being set equal to 0.40 $\times$ the voltage difference causing breakdown in that segment. 164 statistical tries have led to 4482 discharged links. The growth is very sparse or constricted for a considerable distance away from the needle tip.

served type of streamer(types $2,3,4$ [7]) with a different electron-transport mode.

In search of a microscopic origin for these streamershaping effects, we call attention to a model for the microscopic growing tip of streamer filaments, which was proposed by D'yakonov and Kacharovskii [16]for streamers in semiconductors, and has been extended by Atrashev [17] for liquids. At present, this model considers physical parameters which may determine the tip-radius (roughly 3 micrometers) and speed of advance of the individual filament, by affecting the size and shape of the high-electric-field breakdown zone (presumably a region of impact ionization). Future extensions of their model might include consideration of a preference for lateral spreading versus axial concentration of growth, at the microscopic level of the streamer-liead radius.

\section{Programming methods}

Keeping track of the voltage field surrounding the streamer tree, which is a growing highly irregular object, poses the major difficulty. We re-evaluate the voltage field at each growth stage, throughout 

the full interior volume. The enormous flow of numerical activity is handled by parallel (SPMD) computing methods.

We have developed a scalable block-parallel program to realize this model. It exploits the power and local memory of multiprocessors to handle the bulky Laplacian calculation. Each process is assigned a rectangular slab subdivision of the cube volume. Data is passed through the face-planes between adjoining blocks. Details are given in earlier articles $[18,19,20]$.

To simplify programming, we adopt Fortran 90 as the high-level language, taking advantage of its excellent variety of array-directed commands. The physics is made clear by writing the code as if for a single serial processor, but addressing each instruction to large arrays, which include the entire volume of dielectric fluid (for the Laplacian calculation) or the complete group of neighbor sites which must be examined at each growth stage. Because it is fully scalable, the code may be run interactively at small sizes (say $48 \times 48 \times 48$ ) with three processes, then scaled up to 128 -cubed size, with eight processes for a full-sized run.

As in conventional Fortran programming, the arithmetic operations are simplified through intermediate temporary variables, which in this case become very large arrays. All of this is made practical by the large amounts of random access memory (RAM) available to individual processes, on modern multiprocessors. Typically, our code, running on four processors can require from 100 to 200 Mbyte of RAM per processor. Often, in physical problems, it is memory accesses rather than simple computation time which limits the extent of detail that can be obtained.

The program CADMUS is written in a readable high-level language, which leaves the physics clear. Fortran 90 is supplemented by NIST's DPARLIB, a set of subroutines which extend Fortran 90 instructions across block-process boundaries [21], in the environment of the Message Passing Interface (MPI) [22]. It has run on networks of workstations, in the LAM environment [23], but is faster on more tightly coupled multiprocessors, such as the IBM SP2 and SGI Origin $2000^{*}$.

\footnotetext{
${ }^{*}$ Certain commercial equipment and software may be identified in order to adequately specify or describe the subject matter of this work. In no case does such identification imply recommendation or endorsement by the Nationa! Institute of Standards and Technology, nor does it imply that the
}

We offer the source code for interested users, via Internet:

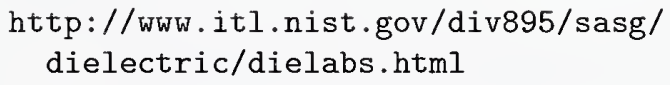

\section{Acknowledgments}

The authors have received assistance from many NIST colleagues. Francis Sullivan brought statistical techniques which have had extensive use in molecular mechanics. Jim Sims and Steve Satterfield have supplied key forms of help with programming and with workstation maintenance. Ken Stricklett provided references and helpful critical review. H.A. Fowler is indebted to the NIST Information Technology Laboratory for access and guestresearcher technical privileges.

This paper is partly based upon a presentation given at the 1999 Conference on Electrical Insulation and Dielectric Phenomena, Austin, TX, Oct.17-20, 1999.

equipment or software is necessarily the best available for the purpose. 



\section{References}

[1] L. Pietronero and H.J. Wiesmann, Z. Phys. B70, 87 (1988). See also S. Satpathy, in Fractals in Physics, L. Pietronero and E. Tosati, eds, Elsevier 1986, pp. 173-176, and A. Erzan, L. Pietronero, and A. Vespignani, Rev. Mod. Phys., 67, no. 3, pp. 545-604 (1995).

[2] P. Biller, Proc. 1993 Int. Conf. on Conduction and Breakdown in Diel. Liquids, Baden-Dätwill, Switzerland, July 19-23, 1993, pp. 199-208.

[3] L.A. Dissado, S.J. Dodd, J.V. Champion, P.I Williams, and J.M. Allison, IEEE Trans. on Diel. and Elect. Ins. 4, no.3 (June 1997) pp. 259-279.

[4] I. Beichl and F.E. Sullivan, "(Monte Carlo) Time after Time", Computational Science and Engineering 4, no. 3 (July-Sept. 1997), pp. 91-94. See also A.B. Bortz, M.H. Kalos, and J.L. Lebowitz, J. Comput. Phys., 17, 10 (1975), especially Appendix B.

[5] H.J. Wiesmann and H.J. Zeller, J. Appl. Phys., 60, no.5 (1986), pp. 1770-1773, esp. Fig. 5.

[6] K. Miyano, K. Yamazawa, M. Pompili, C. Mazzetti, and H. Yamashita, in 1997 Ann. Rept. of the Conf on Elect. Ins. and Diel. Phen., Minneapolis, Oct.1922, 1997, v. II, pp. 640-643, esp. Figures 3 and 4.

[7] R.E. Hebner, in The liquid state and its electrical properties, NATO ASI Series, vol. B193, Plenum Press.

[8] G. Massala and O. Lesaint, in 1997 Ann. Rept. of the Conf. on Elect. Ins. and Diel. Phenomena, Miineapolis, Oct. 19-22, 1997, v. II, pp. 676-679, esp. Fig.1.

[9] O. Lesaint and G. Massala, IEEE Trans. on Diel. and Elec. Ins., 5, no.3 (June 1998), pp. 360-370, esp. Figure 6.

[10] K.L. Stricklett, E.F. Kelley, H. Yamashita, H. Kawai, and C. Fenimore in Conference Record, IEEE Int'l. Symposium on Electrical Insulation, 1990, pp. 161-164.

[11] R. Badent, K. Kist, and A.J. Schwab, Conf. Record of the 1994 IEEE Symposium on Electrical Insulation, Pittsburgh, June 5-8, 1994, pp. 402-405.

[12] W.G. Chadband, IEEE Trans. on Elect. Ins., 23, no. 4, 697 (1988).

[13] G. Massala and O. Lesaint, IEEE Trans on Diel. and Elect.Insulation, 5, no.3, (June 1998), pp.371381 .
[14] D.I. Karpov and A.L. Kupershtokh, Conf. Record of the 1998 Int. Symp. on Elect. Ins., Arlington, VA, USA, June 7-10, 1998, pp.607-610.

[15] R. Badent, Ann. Report of the 1999 Conference on Electrical Insulation and Dielectric Phenomena, Austin, TX, Oct 17-20, 1999, pp. 447-450.

[16] M.I. D'yakonov and V.Yu. Kachorovskii, Sov. Phys. JETP, 67, 5 pp. 1049-1054 (May 1988).

[17] V.M. Atrashev, Proc. 1993 IEEE 11th International Conference on Conduction and Breakdown in Dielectric Liquids (ICDL), Baden-Dättwill, Switzerland, July 19-23, 1993, pp. 264-267.

[18] H.A. Fowler, J.E. Devaney, J.G. Hagedorn, and F.E. Sullivan, Computers in Physics, 12, no.5, pp. 478-487 (1998).

[19] H.A. Fowler, J.E. Devaney, and J.G. Hagedorn, NISTIR 6180, June 1998, "User Guide to CADMUS, a simplified parallel code for Laplacianfractal growth".

[20] H.A. Fowler, J.E. Devaney, and J.G. Hagedorn, Ann. Report of the 1999 Conference on Electrical Insulation and Dielectric Phenomena, Austin, TX, Oct 17-20, 1999, pp.132-136.

[21] John Hagedorn and Alan Heckert, "DPARLIB User's Guide", draft of NIST software library documentation, March 18, 1997.

[22] W. Gropp, E. Lusk, and A. Skjellum, Using MPI, Cambridge (MIT Press), 1994, esp pp. 60-80.

[23] D. Burns and R.B. Daoud, "LAM: an open cluster environment for MPI", in Supercomputing Symposium '94, June 1994, Toronto, Canada. 


